\title{
Estudio del sistema compositivo de Luis Bedmar Encinas
}

\section{Estudo do sistema composição de Luis Bedmar Encinas}

\section{Study of the compositional system by Luis Bedmar Encinas}

\author{
iD Andrés Carlos Manchado López ${ }^{1}$ \\ Conservatorio Superior de Música "Manuel Castillo" de Sevilla, Andalucia, España \\ acmanchado@gmail.com
}

\begin{abstract}
Resumen: Este trabajo es un estudio de la vida profesional de Luis Bedmar Encinas, compositor, director de orquesta y pedagogo musical español, mediante una óptica antropológico-musicológica. A través del estudio de su obra Suite Poliserial para Órgano, se analizará su método compositivo y se mostraran las características más relevantes de su música con el objetivo de demonstrar el desarrollo de un sistema compositivo propio y el conseguimiento de una estética propia - fruto de la unión de la raíz popular del folclore andaluz con los principios estructuralistas utilizados por Schoenberg - que se interna en la expresión de un andalucismo musical profundo y no cae en los tópicos tradicionales ni en imitaciones de lo ya realizado.
\end{abstract}

Palabras clave: Música. Método compositivo. Análisis. Serialismo.

Resumo: Este trabalho é um estudo da vida profissional de Luis Bedmar Encinas, compositor, maestro e pedagogo musical, espanhol, mediante uma óptica antropológico-musicológica. Através do estudo da Suíte Poliserial para Órgano se analisará seu método compositivo e se mostrarão as características mais relevantes de sua música com o objetivo de demonstrar o desenvolvimento de um sistema compositivo próprio e a obtenção de uma estética própria - fruto da união dessa raiz

1 Catedrático del Conservatorio Superior de Música de Sevilla. Academico correspondiente en Sevilla de la Real Academia de Ciencias, Bellas Letras y Nobles Artes de Córdoba, España. 
popular do folclore andaluz com os princípios estruturalistas utilizados por Schoenberg - que se interna na expressão de um andalucismo musical profundo e não cai nos clichés tradicionais nem em imitações já realizadas.

Palavras-chaves: Música. Método de composição. Análise. Serialismo.

Submetido em: 29 de outubro de 2020

Aceito em: 21 de fevereiro de 2021 


\section{Introducción}

El presente trabajo, es un estudio de los aspectos más sustanciales de la producción musical de Luis Bedmar Encinas músico que desarrolla su actividad profesional principalmente en la segunda mitad del siglo pasado en la ciudad de Córdoba, en España - partiendo de una concepción antropológica de la música. A pesar de que la música de Luis Bedmar se conoce en diversos países por los diferentes premios obtenidos - sirvan a modo de ejemplo, el Primer Premio en el Concurso de Composición para autores de países de habla hispana, de la Real Academia de Córdoba (1968), y el Primer Premio en el Concurso de Composición de Misas organizado por el III Congreso Internacional Católico de radiodifusión (Sevilla 1969), entre otros - y por los estrenos realizados de sus obras - a modo de ejemplo baste citar, 5 Microformas en Japón y Holanda y el montaje de Athaeneum por el ballet Nacional de España para su gira internacional por el $25^{\circ}$ aniversario de su fundación, además de que obras suyas se estudian en diferentes centros de enseñanza musical, como sucede con sus Tres Movimientos para Piano Solo que se encuentran dentro del programa de estudios del Conservatorio de Reikiavik en Finlandia - no se ha hallado documentación significativa que aborde el estudio de la figura de Luis Bedmar desde el punto de vista musicológico-antropológico que se propone en este trabajo. En este artículo se explicarán las características más importantes del trabajo musical de Luis Bedmar Encinas haciendo hincapié en los pilares que sostienen su universo sonoro, los cuales son el folclore y su método compositivo, prestando especial atención a la explicación de este, por entender que puede ser de interés para aquellas personas que dentro de la composición buscan nuevos caminos, todavía sin agotar, donde poder encontrar nuevas vías de expresión para plasmar sus ideas musicales, amén de poder comprender mejor la música de Luis Bedmar que transcurre en gran parte por la senda de su método compositivo que aplicó por primera vez en su Suite Poliserial para órgano compuesta y estrenada en 1972. 
El objetivo de este trabajo es profundizar en el conocimiento de la música de Luis Bedmar Encinas a través del análisis antropológico y musicológico de su figura, atendiendo a las características fundamentales de su producción musical, ahondando en el estudio de su método compositivo.

La metodología empleada para la realización de este trabajo es una metodología mixta. De hecho ha sido necesario combinar la análisis documental de fuentes impresas variadas - que van a ser desde Prensa, Entrevistas, Iconográficas, Hemerográficas, Artículos de revistas especializadas, Programas de conciertos y Bibliografía (Blacking, 1974; Merriam, 1964; Schoenberg, 1950; Chomsky, 1968; Sausurre, 1916; Schenker, 1990; García Manzano, 2002) - con el método de la entrevista según la historiografía oral actual que tiene su origen en la antropología, en las culturas ágrafas, y se extiende a todos los campos de la historiografía. En la elaboración de este trabajo ha sido fundamental la colaboración del propio Luis Bedmar, a través de multitud de entrevistas. A la hora de abordar el estado de la cuestión se ha planteado una metodología concéntrica: inicialmente esta investigación se centra en averiguar si este tema ha sido tratado como tal a nivel internacional para después proceder a buscar datos a nivel nacional $y$, por último, finalizar la búsqueda centrándose en Córdoba.

\section{Bases Científicas}

El concepto según el cual entre música y sociedad hay una relación es una idea que surge en el ámbito de la etnomusicología gracias a Alan Merriam (1964), antropólogo, que afirma que la etnomusicología puede ser abordada desde dos puntos de vista: el punto de vista antropológico y el punto de vista musicológico. Según Merriam sería ideal hacer una fusión entre la perspectiva musicológica (estudio del repertorio y del producto musical) y la antropológica (estudio de todos aquellos aspectos del comportamiento humano relacionados con la producción musical) 
porque considera necesario estudiar la música en relación a los comportamientos de quien la produce y a sus relaciones con la cultura. De hecho la música tiene su origen en la dimensión comunicativa, siendo un lenguaje y un comportamiento humano, y tal dimensión se produce y se desarrolla en la sociedad, el entorno en el cual el género humano interactúa y comunica. Es así, como recoge Maurizio Disoteo en su libro Antropologia della Musica per Educatori (2001), que se demuestra necesario, además del estudio del repertorio, el estudio de los comportamientos humanos que están en la base de la producción musical para comprender realmente la naturaleza de la música. En la misma línea está el pensamiento de Blacking (1974) que profundiza algunos aspectos examinados por Merriam. Intenta definir cuáles son las bases de la musicalidad humana y considera que para alcanzar este objetivo la investigación tiene que descubrir lo que hay debajo del producto musical (lo que él define como forma audible de la música). Blacking considera el hombre como un "music-maker", integrado en su contexto socio-cultural. En tal perspectiva, Blacking llega a afirmar que toda la música es música popular (en el sentido de 'producto del pueblo') por el hecho de no poder tener sentido fuera de las relaciones sociales, haciendo así decaer la barrera entre música culta y popular.

En cuanto al punto de vista musicológico se refiere, el análisis musical que el estudio propone se realiza desde dos puntos de vista: la línea de pensamiento de Schoenberg (1950) y la de Schenker (1990).

El punto de vista aportado por Schoenberg, en línea con la lingüística estructural de Sausurre (1916) y con la semiótica - según las cuales los signos pueden conformar un lenguaje permitiendo la comunicación - sostiene que la música es un lenguaje autónomo y su evolución depende de la relación entre los elementos que la conforman: 
Una tríada aislada está completamente indefinida en su significado armónico; puede ser la tónica de una tonalidad o un grado de otras varias. La adición de una o más triadas puede restringir su significado a un número menor de tonalidades. Cierto orden confiere a tal sucesión de acordes la función de progresión...Una progresión tiene la función de establecer o contradecir una tonalidad. (Schoenberg, 1990: 15)

En esta línea coincide también con el pensamiento de Umberto Eco: "Una concepción del arte como hacer, hacer concreto, empírico, industrial, en un contexto de elementos materiales y técnicos: un concepto del fenómeno artístico como organismo regido por toda una legalidad estructural..." (Eco, 1987: 15-16).

La otra óptica que complementa este punto de vista es la visión de Schenker (1990) que, inspirado en el pensamiento de Husserl, plantea que hay que llegar a la esencia de las cosas (eidética) y propone la reducción trascendental y el análisis intencional para explicar el sentido del mundo y de las cosas. Schenker parte de esta búsqueda de las estructuras originarias, a partir de la que se crea cada página musical: estructuras profundas que conforman el lenguaje musical. Este concepto se relaciona modernamente con la lingüística generativa de Chomsky (1968) que distingue entre estructuras profundas, o sea la interiorización que el oyente tiene de la lengua, y estructuras superficiales, o sea las estructuras de la realización que el oyente tiene de la misma.

\section{Breves notas biograficas}

Antes de adentrarnos en el estudio del método compositivo así como en otras cuestiones referentes a la obra de Luis Bedmar Encinas es necesario acercarnos un poco a su persona, aportando algunos datos biográficos que nos ayudarán a comprender un poco más la relevancia de este músico español, nacido en Cúllar Baza, provincia de Granada, en 1932, que a los 19 años se traslada 
a Córdoba, ciudad en la que desarrollará su actividad profesional. Estudia en el Conservatorio Superior de Música de Córdoba, terminando con las máximas calificaciones y primeros premios por unanimidad en Solfeo, Armonía y Composición. El trabajo con profesores españoles de reconocido prestigio como Rodolfo Halffter, Cristóbal Halffter, o Miguel Querol, y con profesores extranjeros como el compositor y director de orquesta italiano Franco Donatoni, le proporciona un conocimiento amplio de las vanguardias musicales del momento en España y Europa; especial mención merece su amistad con Gerardo Gombáu con el que compartirá opiniones y del que recibirá importantes consejos a lo largo de los años. Fue profesor de Solfeo, Armonía y Conjunto Coral e Instrumental del Conservatorio Superior de Música de Córdoba y Director de la Orquesta de Cámara del mismo. Desde el conservatorio ejerció una gran labor pedagógica sobre muchas generaciones de alumnos, a los que transmitió su gran interés por la música andaluza y cordobesa. Su trabajo como compositor se ha visto reconocido con la obtención de diferentes galardones a nivel nacional e internacional.

En 1974 fue nombrado Director de la Banda Municipal de Córdoba y desde esta posición impulsó su conversión en la que fue Orquesta Municipal de Ciudad de Córdoba. Diversas obras suyas han sido estrenadas por la Orquesta de Córdoba, entre las que destacan Sueño de Córdoba, la Sinfonía de las Tres Culturas o el Concierto para guitarra y orquesta, estrenado bajo la dirección de Leo Brower. Así mismo la Cantata del Albaicín, fue estrenada por la Orquesta de Granada y el Coro de la Federación Granadina de Coros. Ha realizado un gran número de grabaciones, tanto de música propia como de otros compositores, dirigiendo la Banda Municipal de Córdoba, la Orquesta Municipal de Ciudad de Córdoba y la Coral de la Cátedra Ramón Medina y más de medio centenar de sus composiciones han sido estrenadas y grabadas en disco. Algunas de estas composiciones, como $A$ la fuente del Olivo, figuran en el repertorio habitual de gran parte de los coros españoles, habiéndose interpretado también en varios países de Hispanoamérica y Estados Unidos. 
Ha recibido homenajes de la actual Orquesta de Córdoba, Orquesta de Cámara Manuel de Falla de la Diputación de Cádiz, del Ateneo de Córdoba, así como distinciones honoríficas de varios ayuntamientos y entidades de Andalucía, destacando su nombramiento de Académico Numerario de la Real Academia de Córdoba de Ciencias, Bellas Letras y Nobles Artes.

\section{Contexto y estilo}

Antes de adentrarnos en el estudio propuesto sobre la música de Luis Bedmar Encinas, es conveniente una breve contextualización histórico-musical. En los primeros veinte años del siglo, la música romántica pasó por un feliz momento de creación y aprobación. Los exponentes más valiosos fueron Giacomo Puccini y Richard Strauss; de la misma manera fueron Claude Debussy y Gustav Mahler, pero las composiciones de estos marcaron el final de las concepciones románticas, llevando en sí mismos los gérmenes de la crisis del sistema armónico-tonal que había constituido la música a partir de 1700 en adelante. Los compositores más importantes y representativos de esta época, empezando por Ravel, contribuyeron a superar la concepción romántica. Los ataques a la popularidad de la tonalidad clásica llegaban de varias direcciones con la ampliación del área de la disonancia (Stravinskij, Prokofiev, Hindemith). En algunos casos la renovación fue fruto de una liberación espiritual (Skrjabin) y técnica y fue buscada a través de distintas vías: el uso de escalas deducidas del estudio del canto popular (Bartók) o construidas especialmente con la utilización de intervalos más pequeños que el semitono (Busoni, Hába). El hecho más radical fue la anulación en la atonalidad de cualquier jerarquía entre sonidos y de todos los nexos entre acordes y el consiguiente establecimiento de una organización de los sonidos con el uso de las doce notas de la octava cromática: la dodecafonía (Schoenberg, Berg, Webern). 
El radicalismo atonal de la Escuela de Viena encontró grandes dificultades de expansión, en un primer momento por las objetivas dificultades de aceptación y comprensión por parte del público; después del 1933 también como consecuencia de la oposición del nazismo al "arte degenerado", en Alemania. Muchos compositores habían retomado la escritura de composiciones menos ásperas y más relajadas. Se difundía la orientación hacía un nuevo clasicismo (neo-clasicismo), donde la recuperación de las formas del pasado permitía posiciones "objetivas"; fueron abolidos los psicologismos y las posiciones sentimentales; a la idea de música como expresión se fue sustituyendo por la de la música como construcción. En esta posición convergieron muchos compositores europeos. Entre los principales: Satie, Ravel, Milhaud, Poulenc, Hindemith, Malipiero, Casella, Bartók, Kodály, Falla; pero especialmente Stravinskij, que había abierto aquel camino a partir de 1920 (Pulcinella) y propuesto también la fórmula de la "música al cuadrado", que consistía en actuar (o trabajar) sobre textos o giros característicos de músicos de los pasados siglos. Entre el 1935 y el 1940 muchos compositores, entre los cuales Schoenberg, Stravinskij, Bartók, Hindemith y Milhaud, se trasladaron en Estados Unidos. La segunda guerra mundial (1939-1945) marcó la separación clara entre las generaciones que había operado en la primera mitad del siglo y los músicos nacidos después el 1920. Muchos de estos últimos adquirieron una orientación teórico-práctica común, cursando, a partir de 1950, los Cursos de verano de Darmstadt. El punto de partida de estos cursos - también gracias a la acción de divulgación empezada por René Leibowitz con los escritos Schoenberg et son école (1946) y la Introduction a la musique de douze sons (1949), y con las execuciones - fue la experiencia dodecafónica, profundizada a través de la obra de Webern más que a través de la enseñanza de Schoenberg. Webern fue entendido como el estímulo, la ocasión para extender el principio del serialismo dodecafónico y otros elementos o parámetros del sonido: la altura, el ritmo, la durada, el timbre, la dinámica y así adelante. Este rigorismo envolvió en distintas maneras los compositores que se habían formado a 
Damstadt: Maderna, Boulez, Nono, Stockhausen, Berio, Pousseur, Ligeti, entre otros.

Este fue el punto de partida, no de llegada, de la Nueva Musica que, desarrollándose en el sentido de profundizar mayormente los aspectos constructivos y constitutivos de la materia sonora, consiguió las metas opuestas en la cuales prevalían la libertad, el non-rigor, hasta la introducción dell' alea, que permitía a los ejecutores posibilidades de elecciones o intervenciones también determinantes en el texto escrito o en partes de ello. (Allorto, 2005).

En este contexto musical es donde se desarrolla la actividad compositiva de Luis Bedmar Encinas, músico que denominaremos poliédrico, porque a lo largo de su vasta obra musical podemos encontrar diferentes modos de llevar a cabo la composición musical. Su amplia producción (más de 300 obras propias, arreglos corales e instrumentaciones) abarca desde el género sinfónico, coral, hasta obras de música de cámara. Estructuralmente aborda multitud de formas y armónicamente se mueve partiendo desde un arcaísmo primitivista - tomando algunos motivos e influencias desde la música del primer siglo -, pasando por el gregoriano, haciendo un estudio muy profundo de la estética renacentista, asimilando incluso procedimientos compositivos del barroco y un conocimiento claro de las armonías clásicas y utilizando materiales sonoros de todas las épocas hasta nuestros días. Una primera característica que hay que tener en cuenta a la hora de acercarse a la música de Luis Bedmar es el profundo conocimiento que atesora de la música popular: buena parte de su trabajo la ha dedicado a la recuperación y puesta en valor del Patrimonio Musical cordobés y andaluz y fruto de este trabajo ha sido la publicación de diversos libros destacando Música Popular de Córdoba y su Provincia (Bedmar Encinas, 2008). Sus investigaciones en este campo, siguiendo el camino marcado por Bela Bartok y Zoltan Kodaly en el estudio y la investigación del canto popular en Hungría, le permiten captar de una manera bien precisa la esencia de la música andaluza: esto le dará una impronta muy característica a su música, impronta que desarrollará a lo largo de su dilatada experiencia profesional. 
En este punto hay que resaltar la coherencia personal y estética de Luis Bedmar, al ser un músico que desde sus inicios hasta nuestros días ha cultivado el estudio y profundizado en la investigación etnomusicológica del folclore andaluz llegando a captar su esencia y consiguiendo por un lado llegar a una abstracción perfecta y eidética de lo andaluz - que le ayuda a generar todo tipo de música con esta esencia - y por otro adaptarse a cada una de las vertientes estilísticas en las que trabaja.

Luis Bedmar muestra a lo largo de su obra como abordar la composición de la canción popular y su armonización: viene a colación decir que un gran número de sus canciones, como $A$ la fuente del Olivo, son consideradas por el pueblo de Córdoba como propias, lo que a su vez nos indica el grado de unión existente entre Luis Bedmar Encinas y la cultura popular cordobesa. Baste en este punto lo ya avanzado, puesto que el estudio pormenorizado de esta cuestión se está llevando a cabo en otro trabajo independiente por lógicos motivos de extensión. Al mismo tiempo, a lo largo de su obra, crea un nuevo lenguaje que, a pesar de su modernidad, continúa unido a la sociedad que le hizo nacer, como recoge J.M. Moreno Calderon en el libro Música y Músicos de la Córdoba Contemporánea (1999: 252):

Luis Bedmar Encinas era ya especialmente conocido como compositor, actividad en la que había obtenido varios premios nacionales y estrenado con éxito diversas obras, sinfónicas y sinfónico-corales, música de cámara, obras para banda, música religiosa y numerosas canciones. Es el caso de La Cantata de las Gracias (1959); Obertura cordobesa (1959), exponente del folclorismo que caracterizará una parte de su obra compositiva posterior.

Dentro de este estilo compositivo de Luis Bedmar también se puede citar la Suite Andaluza para coro a cuatro voces mixtas, que contiene la armonización de ocho canciones populares correspondientes a cada una de las provincias andaluzas. 
A partir de 1972, en que se publica su Suite Poliserial para Órgano, gran parte de su obra se basa en la práctica de varios regímenes de coherencias sonoras. La Suite Poliserial para Órgano fue estrenada en el Conservatorio Superior de Música de Córdoba el 6 de octubre de 1972 y marcará un antes y un después en la trayectoria musical del compositor: "partitura que marcaría el inicio de un estilo propio y que fue estrenada por el eminente compositor y organista granadino Juan Alfonso García". (Moreno Calderón, 1999: 252).

\section{El método y la Suite Poliserial}

Luis Bedmar desarrolla un sistema compositivo que se podría denominar como "serialismo amable": aunque se trate de una denominación no científica, pero se puede constatar que es el resultado que se obtiene al integrar este sistema de composición. A la hora de abordar el estudio de este método llama poderosamente la atención que Luis Bedmar parte de un concepto que es desarrollado a través de un método en el mismo momento en que está componiendo la obra y, a su vez, este es el punto de inicio de una nueva manera de componer que se irá desarrollando en el tiempo. Esta simultaneidad Ilama la atención porque Bedmar, a pesar de idear su método basándose en los conceptos elaborados por Schoenberg, sigue un camino opuesto para su desarrollo.

El propio Schoenberg, en su libro El Estilo y la idea, publicado en 1950, recoge una serie de ensayos sobre temáticas diversas: uno de estos se titula la Composición con doce sonidos y en este escrito de marcado carácter pedagógico describe pormenorizadamente su método de composición dodecafónico, método de importancia capital en el desarrollo de la música en el siglo XX. En 1922, Schoenberg declaraba a Josef Rufer: "He hecho un descubrimiento capaz de asegurar la supremacía de la música alemana durante 100 años". (Stuckenschmidt, 1964: 107). El Estilo y la Idea (1950) supone una reflexión madura acerca del método dodecafónico llevada a cabo por su creador, reflexión que llega después de muchos años 
de experiencia en la utilización del mismo y de un concienzudo trabajo de síntesis y comprensión por parte de Schoenberg, como recoge Stuckenschmidt (1964) en su libro Arnold Schoenberg:

\begin{abstract}
El método de composición con doce sonidos es el fruto de toda una sucesión de experiencias. La primera de ellas se remonta a diciembre de 1914 [...] en el otoño de 1921 [...] me di súbitamente cuenta de la verdadera importancia de hecho al que había llegado inconscientemente. (Stuckenschmidt, 1964: 113-114)
\end{abstract}

Schönberg sostiene que el dodecafonismo surge de una necesidad de creación de un nuevo lenguaje al hacerse evidente que la tonalidad se ha ido diluyendo a lo largo del siglo XIX debido a la extensión del cromatismo y al agotamiento de la composición basada en la creación de tensión y distensión por medio de los encadenamientos armónicos; además la disonancia es aceptada sin límite por el oyente. La emancipación de la disonancia es total y se coloca al mismo nivel de la consonancia. Este sistema genera piezas breves pero de un expresividad total.

Luis Bedmar denomina a su método "poliserialismo" y la plasmación del mismo se lleva a cabo en su Suite Poliserial para Órgano, en donde Luis Bedmar vierte por primera vez y de una manera ordenada las ideas que, referidas a la composición, han surgido en su mente. El procedimiento es consecuencia del deseo de romper la atracción tonal del sistema clásico, sin caer para conseguirlo en el empleo de la disonancia.

A modo contrario, en el siglo XX, se había utilizado la disonancia para romper la atracción tonal. Stravinsky utiliza un diatonismo ampliado, siendo algunos de sus procedimientos característicos la disonancia agregada, los obstinatos, los bloques armónicos contrapuntados. En todos se observa el choque de intervalos disonantes, mediante el cual, el autor avanza sobre nuevos horizontes. El politonalismo, cultivado por Milhaud o sus seguidores, se emancipa de la tonalidad presentando varias de ellas simultáneamente; pero esa simultaneidad es más clara 
cuantos más duros son los choques. Bartók preconiza ya el libre uso de la disonancia, tan reflejada en el empleo de los acordes con tercera mayor y menor. Schoenberg, Alban Berg, Anton Webern, Donatoni, y muchos otros autores posteriores, componen bajo el signo serialista, dodecafónico en mayor o menor grado; pero la presencia de los choques disonantes como justificación de un sistema es más que manifiesto, lo que no se aminora en ninguno de los procedimientos posteriormente usados. Claro está, la disonancia constituyó, de una u otra forma, el trampolín para huir de la atracción tonal. Se ha utilizado tanto, que resulta anticuado llamarlo disonancia; se ha perdido de vista su expresión objetiva, y para muchos es tan subjetiva como el resto del lenguaje musical.

En el noble deseo de huir del contenido musical clásico, se ha huido de la consonancia, confundiéndola con la atracción tonal. Sin embargo, una cosa es la consonancia armónica o melódica y otra la atracción tonal, tal como quedó constituida en el clasicismo. Por los caminos de la canción popular, podemos llegar documentalmente hasta himnos como los de Apolo, procedentes del siglo II A.C., el himno a Helios, el cantar de Seikilos e infinidad de cantos del que puede ser muy representativo el gregoriano y que nos demuestran rotundamente que la consonancia no está encerrada en los límites de las paredes de dos siglos, sino que es connatural al hombre y con él ha vivido desde las primeras manifestaciones melódicas del mismo. Si está justificado el abandono de las cadencias, o la atracción de los reposos frecuentes en la tónica, dominante y subdominante, o sus sustitutivos - que de cierta manera siguen existiendo aun cuando se trate de la "tonalidad fluctuante", cercana a la atonalidad - no está justificado el abandono de la consonancia. Quizás en este sentido, uno de los compositores más cercanos a Luis Bedmar Encinas sea Paul Hindemith. Su sistema de cromatismo diatonizado se acerca en la intención, aunque varía en la realización: Hindemith intenta el libre uso de las doce notas de la escala dentro de una estructura tonal. La intención del sistema de Luis Bedmar, que estamos analizando, es romper la estructura tonal a base de consonancias, o por lo menos, sin usar 
la disonancia como trampolín para librarse de la atracción tonal. Es en esto en lo que radica toda la intencionalidad del sistema.

El sistema poliserial nace de las observaciones anteriores y de un deseo de valorar objetivamente las sonoridades, regresando a la época en la que se huyó de la consonancia, considerándola culpable del sistema cerrado, aun cuando sea más abierto de los que le siguiera. El primer paso seguido por Luis Bedmar fue llevar el sistema a su estructuración más completa. Para eso, consideró conveniente plasmar el sistema en una obra, la Suite Poliserial para Órgano en 5 movimientos - dedicada al amigo y organista Juan Alfonso García -, obra de la que vamos a ocuparnos. Desde el comienzo, pensó en una serie que no va a ser desarrollada a la manera tradicional, en que el orden numérico de la misma promueve su armonización. Obsérvese (Figura 1) la serie del primer movimiento de la Suite y su armonización tal y como se presenta en la entrada de dicha obra.

Figura 1. Suite Poliseral para Órgano, I, compases 1-8.

A Juan Alfonso Garcia

SUITE POLISERIAL

Para Órgano

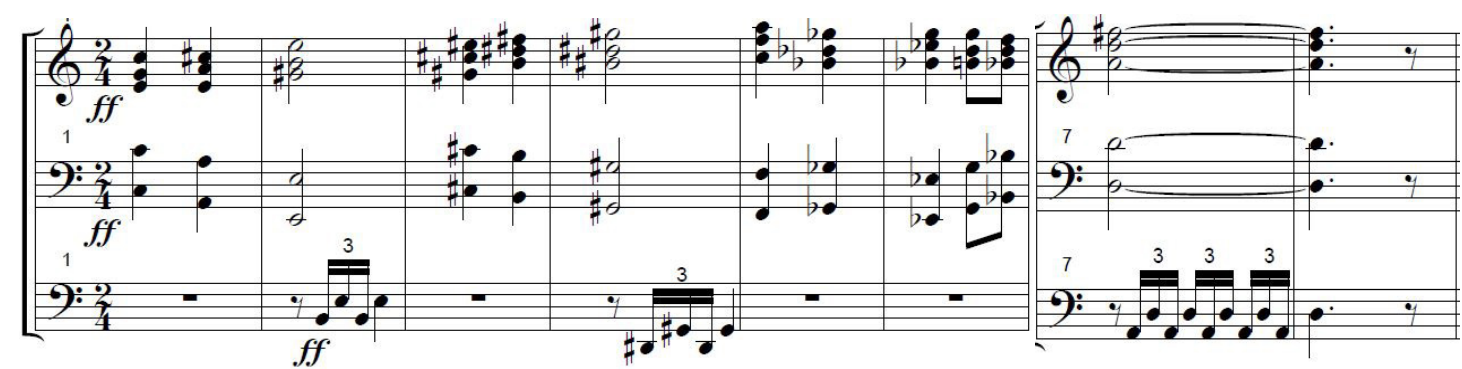

Fuente: Partitura original de Luis Bedmar Encinas

Se trata de una forma distinta de concebir la armonización de la serie. Al ser serie, rompela atracción tonal; y al no existir ésta, se hace posible la organización de todo un bloque de acordes de cualquier constitución (en este caso de acordes prefectos mayores). De esta forma, la sonoridad de este tipo de acordes se prolonga sobre toda la serie, dando un sentido de unidad a la misma, al 
tiempo que produce en su conjunto una sensación prolongada de una sonoridad polarizada en el concepto de brillantez y solemnidad que se consigue entre la elección interválica de la serie y las distribuciones agógicas. Al aplicar el mismo procedimiento al acorde perfecto menor, el resultado que dio fue del máximo rendimiento, quedando demostrados dos hechos. El primero consiste en que la sonoridad puramente subjetiva de este acorde se prolonga durante toda la serie, estableciendo un concepto de unidad a todo lo largo de ella, pero de variedad al mismo tiempo ya que no se repite ningún acorde, a semejanza de lo que ocurre en la serie anterior. El presentar la serie en menor, sobre las mismas notas que la presentada en mayor, es decir sin transportar, produciría una sensación monótona. El segundo hecho - otra circunstancia a sumar - consiste en contrastar con la serie precedente, ya que al constar esta serie de la misma disposición de intervalos en el plano horizontal que la anterior - es decir, al ser la misma serie transportada - se consigue el concepto de variedad, al tiempo que el de unidad o cohesión.

Véase primero cómo quedó organizada la serie armonizada con acordes perfectos menores (Figura 2, compases 9-16).

Figura 2. Suite Poliseral para Órgano, I, compases 7-16.
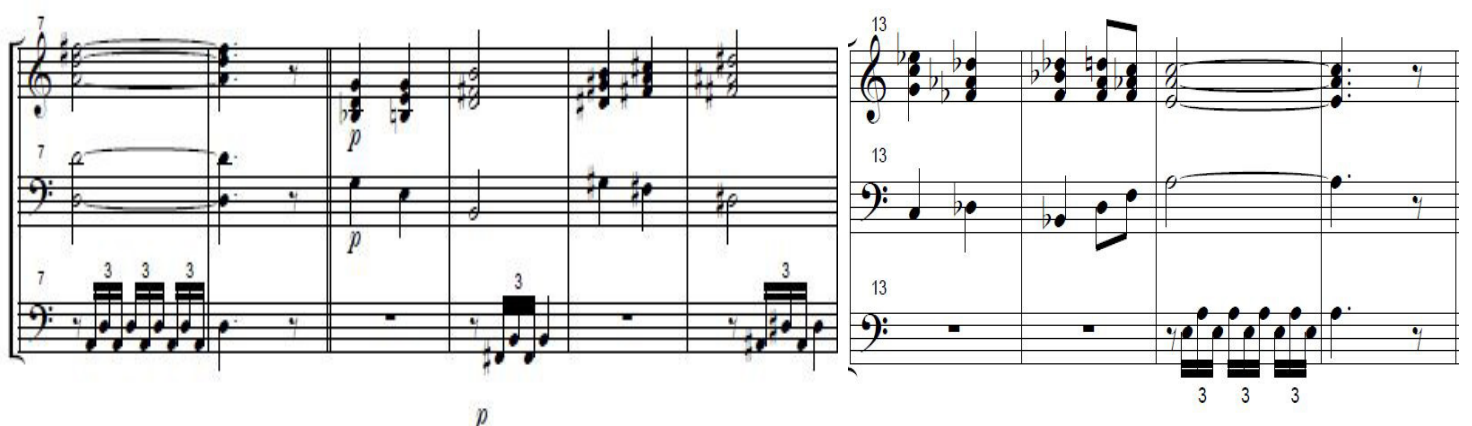

Fuente: Partitura original de Luis Bedmar Encinas

La primera serie, armonizada en mayor, pedía una dinámica y la armonizada en menor exigía la contraria. Si antes se polarizó el concepto de brillantez y de solemnidad, ahora impera contrastante 
la sutileza de un ambiente delicado, exigiendo una mayor agilidad en la agógica y una menor presencia de potencialidad dinámica. Curiosamente se observó que estorbaba la duplicación de las fundamentales en el bajo. No viene el sistema presidido por la matemática de la serie: presenta exigencias artísticas de todo tipo.

Luis Bedmar decidió someter sólo los acordes de tríada al tratamiento poliserial, dejando para más tarde otras combinaciones. De aquí que siguiera la experiencia inmediata con el acorde de quinta disminuida. Al elegir este acorde para acompañar otra versión de la serie, volvieron a hacerse visibles o audibles necesidades de mayor agilización que la observada en la serie en menor. Fue el momento de dar paso a un intento formal, consistente en presentar una métrica que pudiera considerarse como un segundo tema, para volver a repetir la métrica de las anteriores versiones, a manera de tema $A$, que, al serle aplicada la sonoridad del acorde de quinta aumentada, eliminaría toda sensación de monotonía. Véase cómo quedaron estos intentos de ordenación de dos repeticiones más de la serie, armonizadas sucesivamente con acordes de quinta disminuida y quinta aumentada, así como el intento formal al que nos hemos referido (Figura 3, compases 17-30). 
Estudio del sistema compositivo de Luis Bedmar Encinas

Andrés Carlos Manchado López

Figura 3. Suite Poliserial para Órgano, I, compases 13-30.
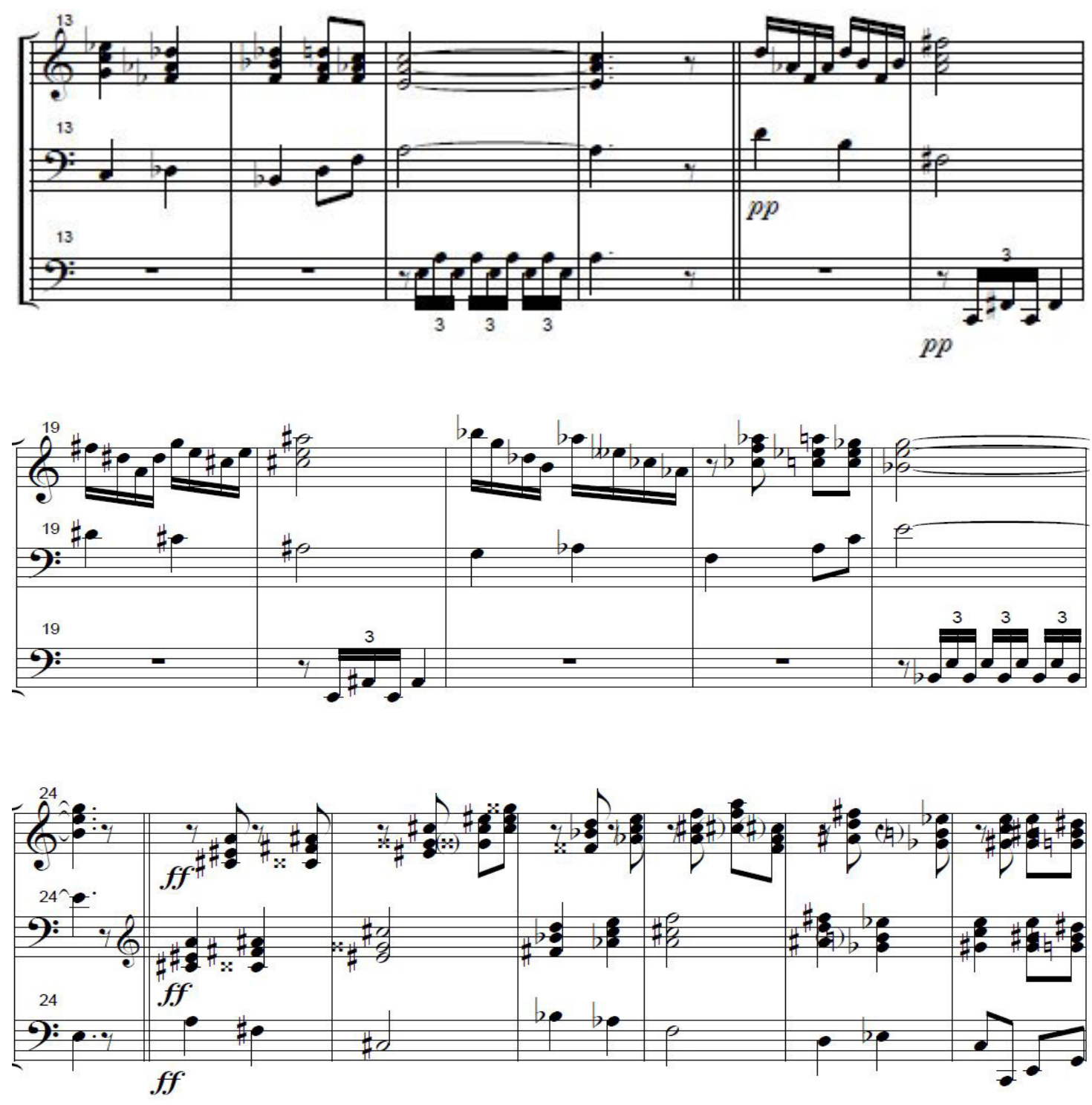

Fuente: Partitura original de Luis Bedmar Encinas

La dinámica de esta serie ha tenido necesidad de reforzarse: sería oportuno discutir si por necesidad formal o necesidad armónica, tal vez por las dos. Pero de ninguna manera quiere decir esto que la serie armonizada con quinta aumentada no pueda presentarse con otro matiz. Sólo en este momento parece necesaria esa dinámica.

Con la posibilidad de intercambiar acordes mayores con menores, se llega a la conclusión de que estos cambios brindan 
grandes posibilidades expresivas, si se hacen de manera ordenada, recurso que Luis Bedmar utiliza en el IV movimiento: este orden consiste en presentar ideas musicales concretas que utilicen varias notas de la serie, formando un pequeño todo (Figura 4).

Figura 4. Suite Poliserial para Órgano, IV. Tiempo de Marcha, compases 41-42.

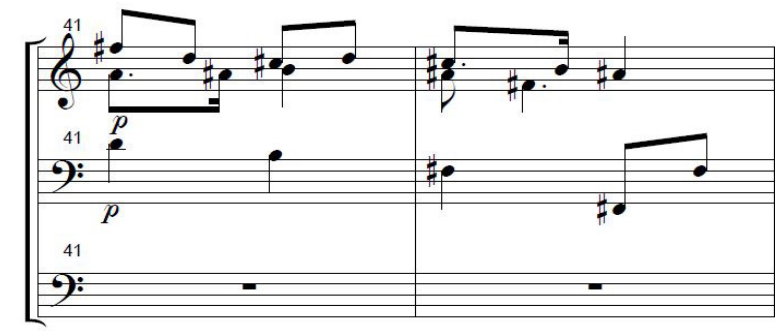

Volviendo al primer movimiento de la Suite, se puede observar como también es posible conseguir un buen efecto con la presentación de todas las sonoridades de los acordes de tríada elementales sobre una sola nota del bajo: así está construido el final de la Entrada (Figura 5, compases 41-44).

Figura 5. Suite Poliserial para Órgano, I, compases 40-44.

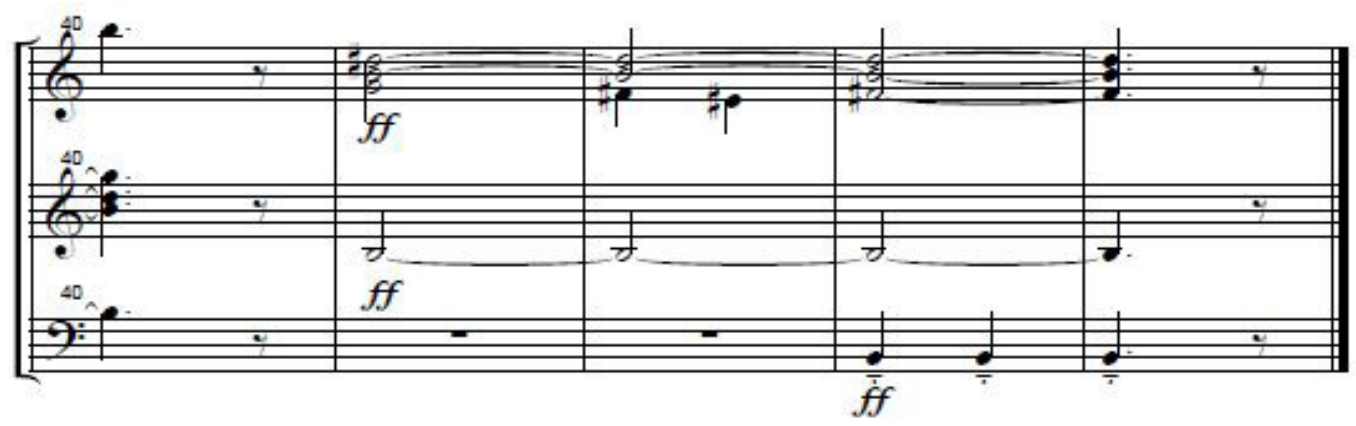

Fuente: Partitura original de Luis Bedmar Encinas

Aquí termina la exposición del sistema en su más acusado sentido armónico: durante toda la Entrada el sistema no ha buscado otra intención que la de su consolidación armónica. 
El tiempo compuesto inmediatamente después fue el Scherzo. Fue el primer intento de sacar consecuencias melódicas al sistema, al mismo tiempo que formales y figurativas, aunque estos dos últimos aspectos estén apuntados ya anteriormente (Figura 6).

Figura 6. Suite Poliserial para Órgano, III. Scherzo, compases 1-5.

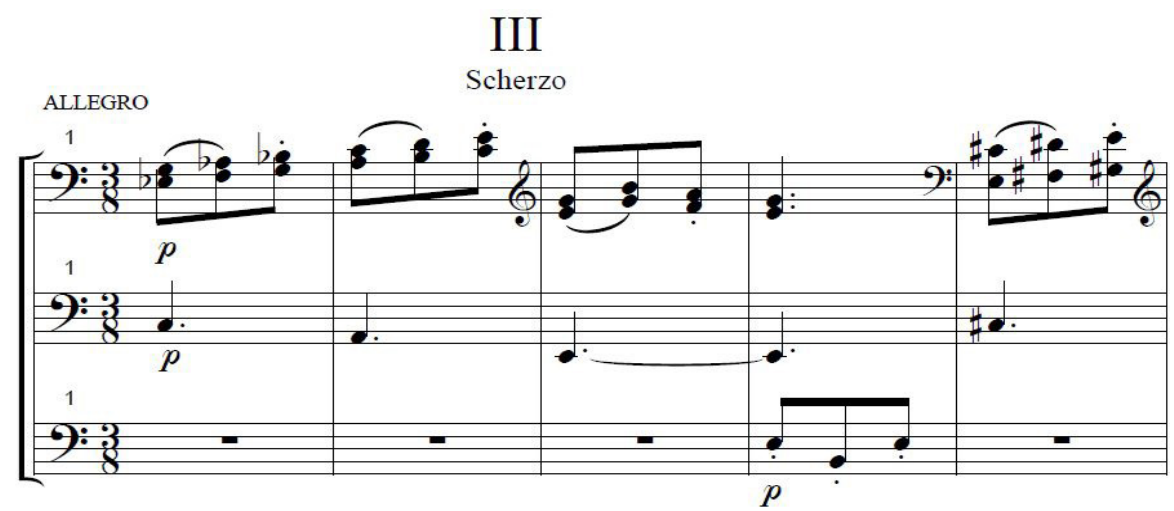

Fuente: Partitura original de Luis Bedmar Encinas

También hemos dicho repetidas veces que se pretendía un sistema que rompiera el círculo tonal clásico, pero utilizando la consonancia. Una cosa es utilizar los más variados procedimientos para hacer audible la disonancia y otra echar mano de este recurso en huida de lo tonal clásico. Otro tanto ha ocurrido con la forma. Entre que a ciertos sistemas no les va bien la forma y que se ha creído que con su utilización se cae en estereotipos, ha progresado paralelamente la anulación de la consonancia con la de lo formal. Así, la música ha ido perdiendo los dos elementos que la hacen más inteligible. No obstante, la forma ha persistido más que la consonancia. A la hora de estructurar el sistema, parece lo más acertado volver a la utilización de lo figurativo. No se debe perder de vista que el arte no tiene sentido si no es comprendido. La música, como todo lenguaje, necesita claridad. Para Luís Bedmar esta claridad o concreción la brinda la forma, no entendiendo por forma el modelo clásico, sino entendiendo por forma todo aquello que se da en la proporción justa y con las repeticiones justas, si las 
necesita, para que la obra sea inteligible por el hombre. Por estas razones, el propio autor se ha refugiado en la forma (para ser más preciso, en la forma de pequeñas proporciones), quedando aclarado que la utilización de la forma, y de cortas dimensiones, no es casual, sino voluntaria (Figura 7) - consecuencia natural si tenemos en cuenta la opinión de Charles Rosen con respecto al serialismo de Schoenberg: "lo que desapareció entre Mozart y Schönberg fue la posibilidad de utilizar grandes bloques de material prefabricado" -. (Rosen, 1983: 31)

Figura 7. Suite Poliserial para Órgano, III. Scherzo, compases 41-44.

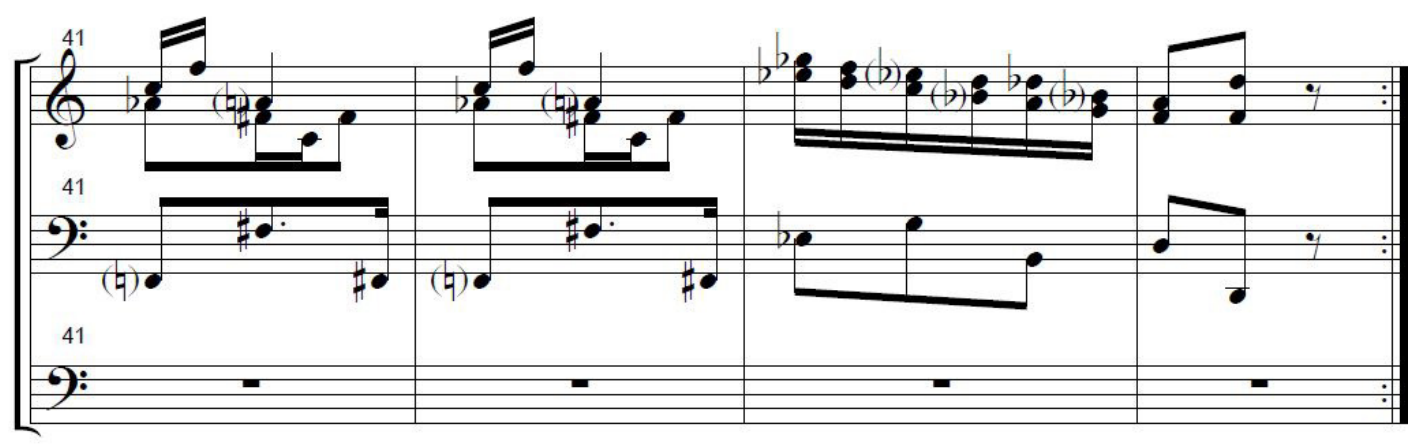

Fuente: Partitura original de Luis Bedmar Encinas

Véase ahora la estructuración melódica del Tiempo de Marcha (Figura 8). Como puede apreciarse, aquí la serie actúa de bajo continuo proporcionando unas armonías que al disolverse se transforman en melodía. En ella intervienen disoluciones armónicas arpegiadas o melodías formadas por notas reales, pertenecientes a la jurisdicción del bajo, así como notas de paso, apoyaturas, floreo, elisiones, etc., alteraciones promovidas por esa especie de microtonalismo, determinado por esta forma de armonizar la serie. El análisis directo de la partitura será más efectivo que las palabras. 
Estudio del sistema compositivo de Luis Bedmar Encinas

Andrés Carlos Manchado López

Figura 8. Suite Poliserial para Órgano. IV. Tiempo de Marcha, compases 1-3.

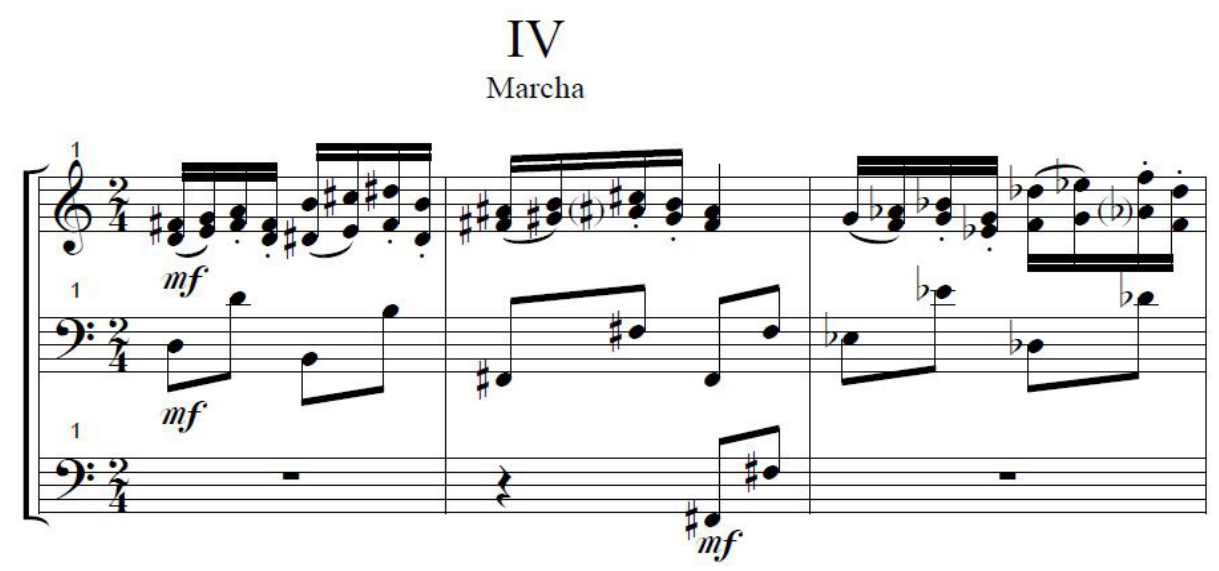

Fuente: Partitura original de Luis Bedmar Encinas

El tema fugado (Figura 9, compases 17-20) no presenta respuesta a la dominante por dos razones fundamentales: la primera porque en este sistema no hay dominante; la segunda, porque después del recorrido de la serie, la nota primera de la serie siguiente es tan nueva como cualquier otra. Pero a Luis Bedmar le brindaba mayor claridad de construcción.

Figura 9. Suite Poliserial para Órgano, IV. Tiempo de Marcha, compases 16-20.
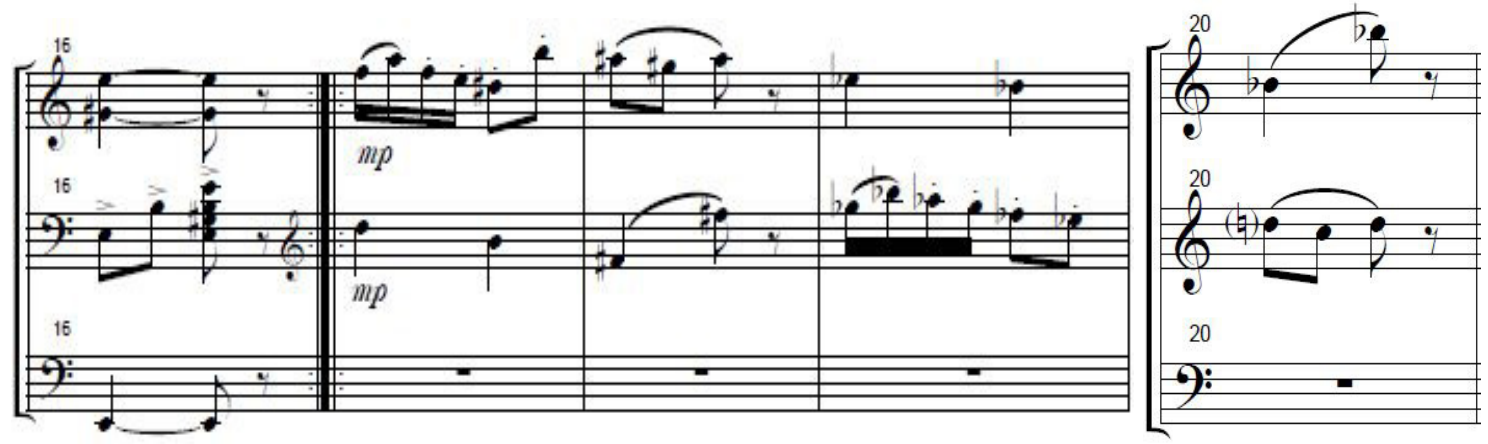

Fuente: Partitura original de Luis Bedmar Encinas

Aquí desaparece ya el uso constante de las fundamentales en el bajo, pasando a la voz a que corresponda la exposición del motivo (que es la serie misma). Así, se empiezan a emplear inversiones de acordes y una manera de hacer el contramotivo 
que llamó la atención a Gerardo Gombau, ya que las armonías que utiliza responden al sentido de microtonalismo, a que antes nos hemos referido (Figura 10).

Figura 10. Suite Poliserial para Órgano, II. Allegretto, compases 1-8.

II
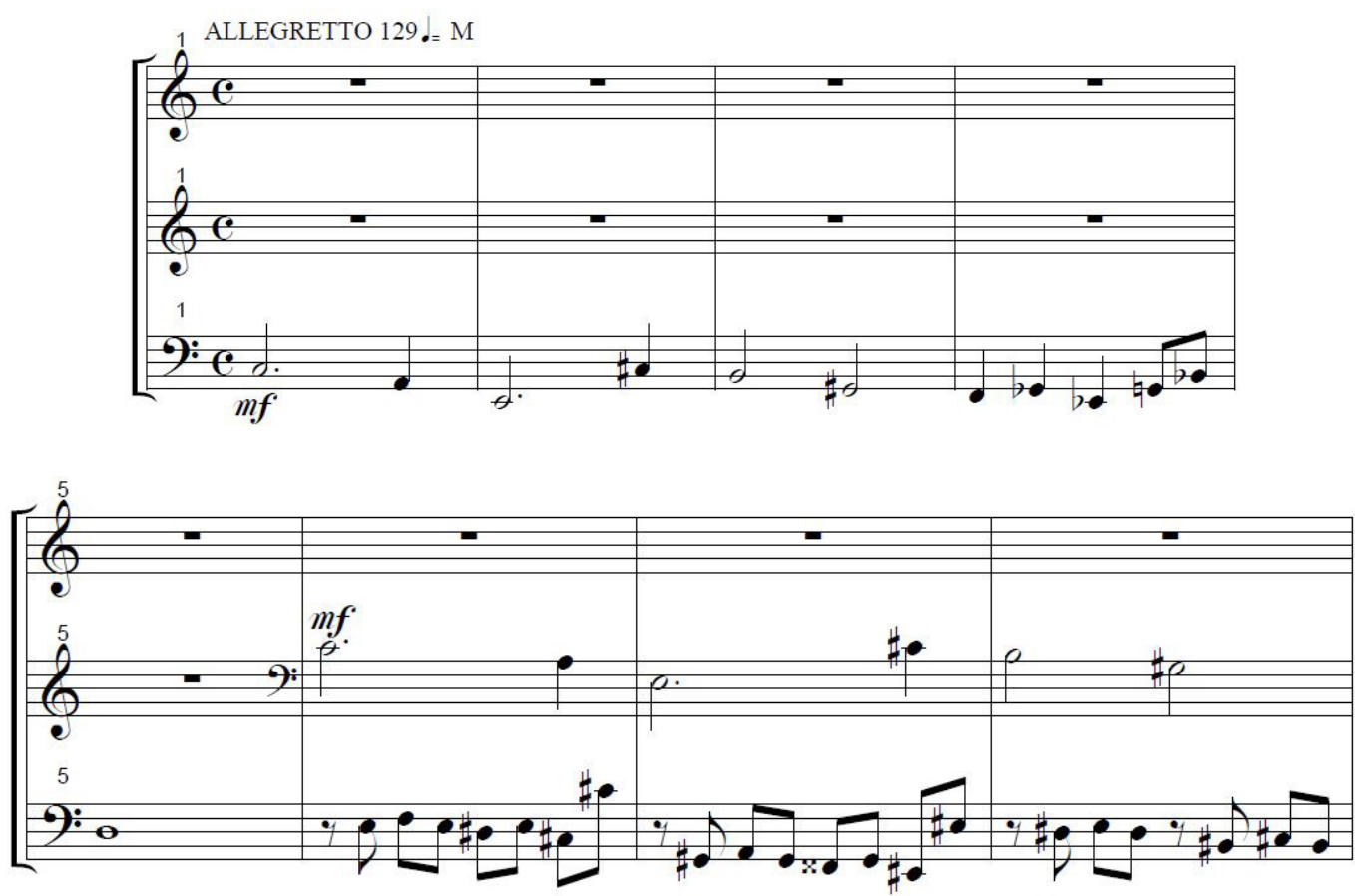

Fuente: Partitura original de Luis Bedmar Encinas

En el plan previsto estaba programado que las fundamentales de los acordes fuesen interpretadas por cualquiera de las partes armónicas. Bedmar había pensado en situaciones más libres, para lo cual era necesario que las notas de la serie quedaran repartidas entre todas las voces. Esto se consiguió tras la exposición a manera de fuga, en un intento de episodio en el que cada voz que hace una imitación, la concluye con la nota correspondiente de la serie.

El episodio termina por dar entrada al tema o serie cuyas armonías representan una serialización de acordes menores, produciéndose al final el principio de la retrogradación que Gerardo Gombau aconsejó a Luis Bedmar. Termina este tiempo después de desarrollarse dicha retrogradación, con un resumen 
armónico de la serie, con la intención de imprimir coherencia por una parte y sensación conclusiva por otra. Para concluir, debía seguir un final más decisivo (Figura 11). Como por otra parte había aspectos contrapuntístico aún inexplorados, el autor se decidió a construir el último tiempo empleando el movimiento contrario del primero. No aporta ya grandes novedades en la elaboración del sistema, ya que, de haber buscado otros recursos, posiblemente se hubiese dañado el sentido general de unidad.

Figura 11. Suite Poliserial para Órgano, V. Final, compases 1-11.
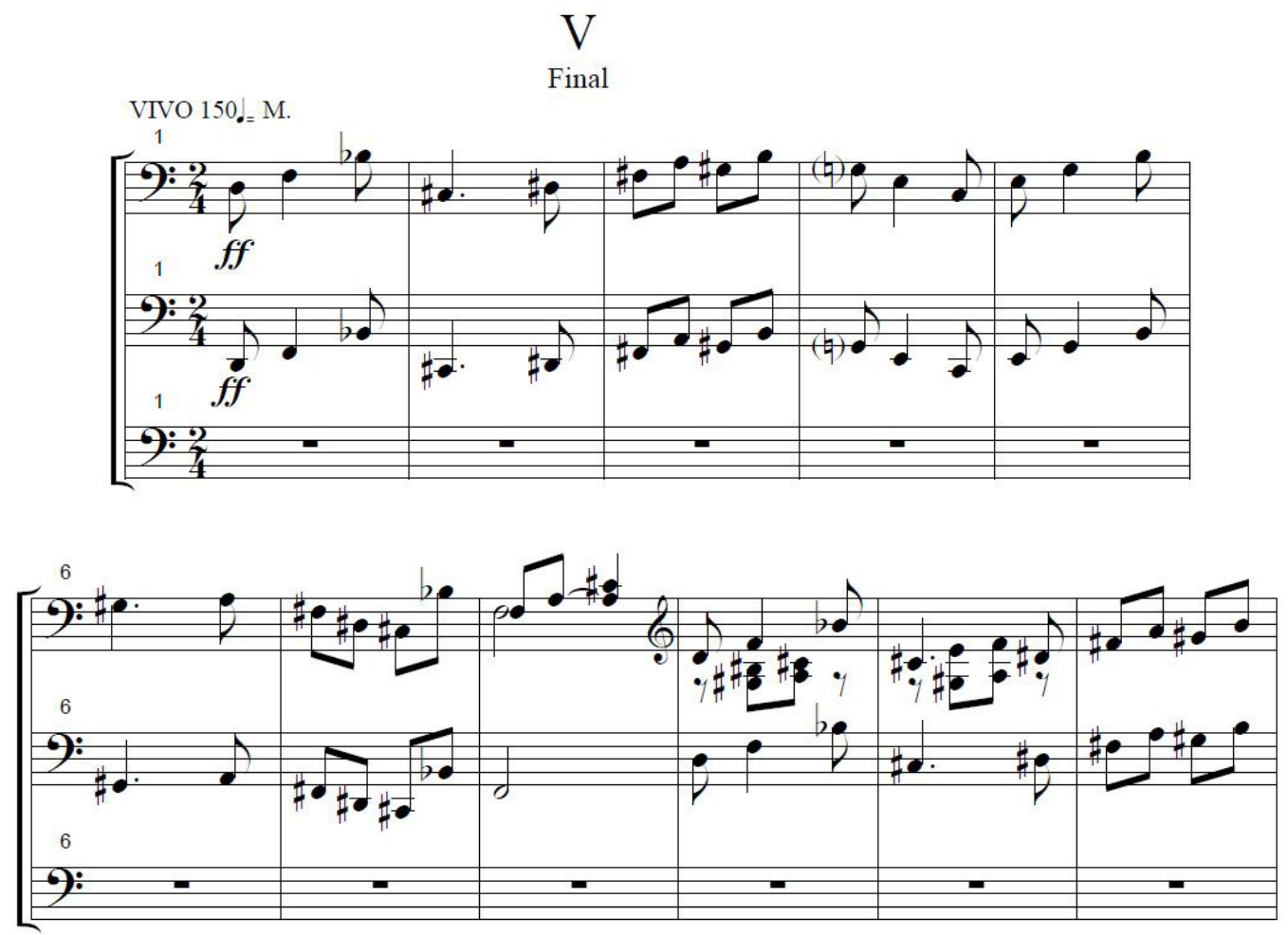

Fuente: Partitura original de Luis Bedmar Encinas

Fruto de la evolución de este método de composición son las obras: Ninfa del Guadalquivir, Crisol, Concierto asturiano y Ateneum. Ninfa del Guadalquivir (1976) y Crisol (1974) son dos pasodobles de concierto, ejemplos de la aplicación del sistema a una forma musical popular típica. El Concierto asturiano (1988), donde Luis Bedmar utiliza tres temas populares del cancionero de Torner 
(obra compuesta para orquesta sinfónica), es una evolución del sistema de Luis Bedmar: partiendo de lo tonal, el tema va presentando transformaciones que van desde el macrotonalismo - cuando el conjunto camina por todos los semitonos de la escala - al microtonalismo, haciendo melodías con los semitonos próximos no cromáticos y utilizando bloques de acordes mayores, menores, aumentados y disminuidos. Ateneum (1990) es un Concierto para Orquesta dividido en tres movimientos, con honda intención andalucista en el cual se emplean diversos regímenes de coherencias sonoras que van desde el microtonalismo al macrotonalismo. En la misma línea están la 2 Sinfonía, la Fantasía andaluza y el Concierto para piano (1974), homenaje a Manuel de Falla, que se internan en un andalucismo musical que intenta ser profundo sin caer en los tópicos tradicionales ni en imitaciones de lo ya realizado.

\section{Conclusiones}

Como se puede observar, la calidad y la cantidad de la obra de Luis Bedmar Encinas son grandes. Luis Bedmar desarrolla un sistema compositivo propio que supone una puerta abierta a músicos de posteriores generaciones. Por todo lo expuesto es posible afirmar que el trabajo compositivo de Luis Bedmar Encinas muestra una coherencia en el concepto artístico propuesto por el compositor al mismo tiempo que abre unos caminos no explorados hasta el momento. Unifica esferas que a priori siempre resultaron contrapuestas: modernidad y arcaísmo, atonalidad y consonancia, serialismo y folclore. En este punto no se puede negar la originalidad de la propuesta artística de Luis Bedmar que partiendo de la raíz más profunda de la música popular - raíz que interioriza de tal manera que se convierte en parte esencial de su producción musical - consigue dotarla de una capacidad expresiva y de una modernidad incontestable aportando líneas argumentales en el campo compositivo que sin duda pueden ser 
aprovechadas por compositores de nuestros días. Se entiende que es un sistema aún por desarrollar por el simple motivo de que Luis Bedmar, como no podía ser de otro modo, solo utiliza una pequeña parte del folclore - la parte correspondiente a Andalucía, y lógicamente no toda -. Luis Bedmar nos hace reflexionar también sobre la cuestión siempre abierta acerca de cúal es el mejor modo de avanzar en música, dejándonos bien claro su consejo: buscar en las raíces más profundas la esencia de las cosas para que de esta manera podamos desarrollar ideas actuales o aplicar modos de hacer contemporáneos sin caer en lo grotesco. La música es un lenguaje que tiene sentido dentro de una sociedad, es un comportamiento humano en una comunidad determinada y necesita del conocimiento de todos los factores que influyen en ese grupo para ser entendida: se puede afirmar que la música de Luis Bedmar participa de esa vinculación con la sociedad cordobesa produciéndose una simbiosis perfecta entre los dos. Bedmar aborda todas las vertientes musicales - música popular, música de banda, coral, sinfónico coral, sinfónica, música de cámara - trabajando formas musicales propias de la cultura andaluza como el pasodoble, las marchas procesionales, música para romerías, canciones, al mismo tiempo que cultiva formas de la llamada música culta como sinfonías, conciertos y cantatas, entre otros, demostrando los planteamientos de Blacking cuando afirma que no hay barreras entre las músicas porque todas nacen del pueblo. Al mismo tiempo Luis Bedmar consigue una estética propia - fruto de la unión de esa raíz popular con los principios estructuralistas utilizados por Schoenberg - que se interna en la expresión de un andalucismo musical que es profundo y no cae en los tópicos tradicionales ni en imitaciones de lo ya realizado. En definitiva, es posible concluir diciendo que Luis Bedmar Encinas es un músico que merece ser estudiado en profundidad y cuya producción musical es merecedora de un lugar de privilegio en la música andaluza del siglo XX. 


\section{Bibliografía}

ALLORTO, Riccardo. Nuova Storia della Musica. Milano: Ricordi, 2005. BEDMAR ENCINAS, Luis. Música Popular de Córdoba y su Provincia. Córdoba: Diputación Provincial de Córdoba, 2008.

BLACKING, John. How musical is man?. Washington: University of Washington Press, 1974.

CHOMSKY, Noam. Language and Mind. New York: Harcourt, Brace Jovanovich, 1972 (Originally, 1968).

DISOTEO, Maurizio. L' antropologia della musica: storia, campi e obiettivi. In: DISOTEO, Maurizio. Antropologia della musica per educatori. Milano: Guerrini e associati, 2001. pp.15-46.

ECO, Umberto. La definición del arte. Barcelona: Ediciones Martínez Roca, 1987.

GARCÍA MANZANO, Julia Esther. Gerardo Gombáu: Época y Obra, 2002, Universidad de Salamanca. Salamanca: Ediciones Universidad de Salamanca, 2002.

GIANNATTASIO, Francesco. II concetto di musica: contributi e prospettive della ricerca etnomusiclogica. Roma: Carocci Editore, 1992.

MERRIAM, Alan P. The Anthropology of Music. Evanston, Illinois: North-western University Press, 1964.

MORENO CALDERON, Juan M. De la banda a la orquesta Ciudad de Córdoba. In: MORENO CALDERON, Juan M. Música y Músicos de la Córdoba contemporánea. Córdoba: Cajasur Editorial, 1999. pp. 251 258.

ROSEN, Charles. Schönberg. Barcelona: Antoni Bosch, 1983.

SAUSURRE, Ferdinand de. Course de Linguistique general. París: Éditions Payot \& Rivages, 1916.

SCHOENBERG, Arnold. Style and idea. New York: Philosophical Library, 1950. 
Estudio del sistema compositivo de Luis Bedmar Encinas

Andrés Carlos Manchado López

SCHOENBERG, Arnold. Funciones estructurales de la armonía.

Barcelona: Ed. Labor, 1990.

SCHENKER, Heinrich. 1990. Schenker studies. Cambridge: Cambridge University Press, 1990.

STUCKENSCHMIDT, Hans. H. Arnold Schönberg. Madrid: Ediciones Rialp, 1964.

\section{Publisher}

Universidade Federal de Goiás. Escola de Música e Artes Cênicas. Programa de Pós-graduação em Música. Publicação no Portal de Periódicos UFG.

As ideias expressadas neste artigo são de responsabilidade de seus autores, não representando, necessariamente, a opinião dos editores ou da universidade. 